\title{
Spectrally and Spatially Resolved Smith-Purcell Radiation in Plasmonic Crystals with Short-Range Disorder
}

\author{
I. Kaminer, ${ }^{1,2, *}$ S. E. Kooi, ${ }^{3}$ R. Shiloh, ${ }^{4}$ B. Zhen,,${ }^{2,5}$ Y. Shen, ${ }^{1}$ J. J. López, ${ }^{1,3,6}$ R. Remez, ${ }^{4}$ S. A. Skirlo, ${ }^{1}$ \\ Y. Yang, ${ }^{1,6}$ J. D. Joannopoulos, ${ }^{1,3}$ A. Arie, ${ }^{4}$ and M. Soljačicic ${ }^{1,2}$ \\ ${ }^{1}$ Department of Physics, Massachusetts Institute of Technology, \\ 77 Massachusetts Avenue, Cambridge, Massachusetts 02139, USA \\ ${ }^{2}$ Research Laboratory of Electronics, Massachusetts Institute of Technology, \\ 77 Massachusetts Avenue, Cambridge, Massachusetts 02139, USA \\ ${ }^{3}$ Institute for Soldier Nanotechnologies, 77 Massachusetts Avenue, \\ Cambridge, Massachusetts 02139, USA \\ ${ }^{4}$ School of Electrical Engineering, Fleischman Faculty of Engineering, \\ Tel-Aviv University, Tel-Aviv 69978, Israel \\ ${ }^{5}$ Physics Department and Solid State Institute, Technion, Haifa 32000, Israel \\ ${ }^{6}$ Department of Electrical Engineering and Computer Science, Massachusetts Institute of Technology, \\ 77 Massachusetts Avenue, Cambridge, Massachusetts 02139, USA
}

(Received 20 July 2016; revised manuscript received 21 October 2016; published 17 January 2017)

\begin{abstract}
Electrons interacting with plasmonic structures can give rise to resonant excitations in localized plasmonic cavities and to collective excitations in periodic structures. We investigate the presence of resonant features and disorder in the conventional Smith-Purcell effect (electrons interacting with periodic structures) and observe the simultaneous excitation of both the plasmonic resonances and the collective excitations. For this purpose, we introduce a new scanning-electron-microscope-based setup that allows us to probe and directly image new features of electron-photon interactions in nanophotonic structures like plasmonic crystals with strong disorder. Our work creates new possibilities for probing nanostructures with free electrons, with potential applications that include tunable sources of short-wavelength radiation and plasmonic-based particle accelerators.
\end{abstract}

DOI: 10.1103/PhysRevX.7.011003

Subject Areas: Optics, Plasmonics

Modern nanofabrication techniques have led to significant advances in the field of photonics, opening new opportunities to study fundamental effects involving the interaction of free electrons with matter and light. For example, there has been a resurgence of interest in the Cherenkov effect [1], which describes the bluish glow emitted from charged particles moving in a medium, with velocity higher than the speed of light in that medium. The Cherenkov effect has recently been found in new materials such as plasmonic surfaces [2], metamaterials [3,4], photonic crystals [5], and even graphene [6,7], exhibiting new phenomena in both the classical and the quantum regime $[8,9]$. In particular, the appearance of the Cherenkov effect in periodic photonic structures demonstrated the fundamental connections $[5,10]$ between the Cherenkov effect and another key phenomenon in electron-photon interactions, called the Smith-Purcell effect [11].

\footnotetext{
* Corresponding author. kaminer@tx.technion.ac.il

Published by the American Physical Society under the terms of the Creative Commons Attribution 3.0 License. Further distribution of this work must maintain attribution to the author(s) and the published article's title, journal citation, and DOI.
}

The conventional Smith-Purcell effect describes light emission by a collective excitation that is induced by a free electron when it couples, through its near field, to the electromagnetic modes of a grating or a periodic structure [10-13]. The Smith-Purcell effect critically depends on precise fabrication of such a periodic structure, which is why the substantial progress in nanofabrication techniques in recent years has revealed a large variety of new applications (e.g., Refs. [14-20]) based on the SmithPurcell effect. Interestingly, the inverse Smith-Purcell effect has also shown exciting possibilities [21,22], which consequently led to more advanced photonic crystal structures being used as promising platforms for particle acceleration by light excitation of a periodic structure [23-25]. In general, photonic crystals have shown a wealth of Smith-Purcell-like effects [5,26-28], with some papers even predicting that photonic crystals will enhance the conventional Smith-Purcell radiation beyond what is possible with regular diffraction gratings [26]. A very recent theoretical work investigated the Smith-Purcell effect in aperiodic structures [29]. Furthermore, other theoretical studies have predicted the enhancement of SmithPurcell radiation arising from plasmonic crystals (e.g., Refs. [30-32]), with recent work (e.g., Ref. [33]) detailing an analytic theory that gives quite good agreement with full 
electromagnetic simulations. Plasmonic structures are of special interest in this context because their interaction with free electrons can, in principle, involve strongly localized resonances in addition to the Smith-Purcell-like collective excitations.

Free electrons have been found to couple very strongly to localized plasmonic resonances. In fact, plasmons were first found as energy loss features in the spectra of electrons reflected from metal surfaces $[34,35]$ due to the interaction of the electrons with the collective oscillation of electron excitations in the metal (a.k.a. plasmons). Over the years, using free electrons has become a significant tool in studying both localized plasmonic resonances and surface plasmon resonances [36-40]. Localized plasmonic resonances often appear because of disorder and roughness on metal surfaces, with surface-enhanced Raman spectroscopy being the most famous example [41,42]. At the same time, the effects of disorder on collective modes in periodic structure have been widely studied through the theory of Anderson localization [43-45]. However, there is no existing theory or experiment studying the effects of disorder on collective excitations of electron-photon interactions, such as the Smith-Purcell effect.

It is the goal of the current paper to study and observe physical phenomena involving localized resonances and effects of disorder coexisting with collective excitations in the coupling of free electrons with modes of light. Current techniques for exact control of free electrons, and, in particular, the precision of electron microscopes, are an optimal tool for simultaneously studying collective phenomena such as the conventional Smith-Purcell effect and localized phenomena such as plasmonic resonances. While these phenomena usually appear in completely different settings, they should occur simultaneously given the proper nanophotonic structure and the proper experimental setup.

Here, we present a new experimental setup based on a scanning electron microscope (SEM) that we modify to allow us to observe the interaction of free electrons moving parallel to the surface of an arbitrary nanophotonic structure such as a photonic crystal slab or periodic plasmonic grating. The setup allows direct optical imaging of the surface while simultaneously allowing the collection of the emitted spectrum. Using this setup, we explore some fundamental aspects of the coupling of free electrons to plasmonic crystals and localized plasmonic resonances. Specifically, we couple the electron beam to onedimensional (1D) line defects in periodic plasmonic structures and observe enhanced and inhibited emissions from such defects, which we can distinguish from the surrounding conventional Smith-Purcell emission through their spectral signature. Then, we measure the Smith-Purcell effect from plasmonic crystals with strong disorder, finding surprising robustness to disorder. There, mixed with the conventional Smith-Purcell spectrum, we find the spectral signature of a localized plasmonic resonance, which we distinguish through polarization filtering. Altogether, our work presents the novel capability of using an SEM to align free electrons passing nearly parallel to a nanophotonic structure, and we retrieve both the spectrum and the spatial information of the light emission. Consequently, we can learn more about the interplay between collective and resonant effects in electron-photon interactions. Such interactions of free electrons with specially designed nanophotonic structures can lead to tunable radiation sources from the terahertz [7], infrared and visible [15], to the x ray [46], as well as provide new sensing and diagnostics tools.

The experimental setup (presented in Fig. 1) is comprised of a conventional SEM with the sample mounted vertical to the stage, having its surface nearly parallel to the electron column. A microscope objective is placed on the SEM stage to collect and image the light emission from the surface. The light collected by the objective passes through a borosilicate viewport and a leaded glass window and is sent through a series of free-space optics that include a beam splitter, allowing simultaneous measurement of the spectrum and the spatial emission pattern. The SEM is operated in spot mode, which we control precisely to align the beam so that it passes tangentially to the surface near the desired area of the sample (in practice, the sample is tilted with a small anticlockwise angle to prevent the beam spread from obscuring the electron path-see Methods section).

We note that a large variety of experimental setups have been used over the years to collect photons emitted from an electron excitation, which is generally called cathodoluminescence (CL) in the electron microscopy community. Many conventional CL setups allow for the spectrally resolved collection of emitted light, some even using proprietary setups for measuring the Smith-Purcell spectra (e.g., Ref. [15]). However, most CL setups are intended for studying interactions of free electrons impinging orthogonally, or nearly so, to a sample that lies approximately flat (focusing the electron beam to a spot and scanning for spatial information). Instead, our setup allows the collection of light emitted from free electrons moving parallel to a surface (see Fig. 1). Moreover, we obtain a direct image of the surface, revealing where the light is emitted from. Such a measurement is particularly interesting for light-electron interactions involving structural defects, or when resolving the dynamics of surface-emitted light. In contrast, other CL setups collect the emitted light with a fiber $[18,47]$ or a parabolic mirror, with the latter allowing angular-resolved CL collection [19,48-51]. Yet, despite these techniques, no direct spatially resolved imaging of the light emission has been performed before, to the best of our knowledge.

To date, angularly resolved CL collection has been particularly useful for measuring local photonic and plasmonic cavities and resonances on a surface, when excited locally by a free electron and then emitting to the 


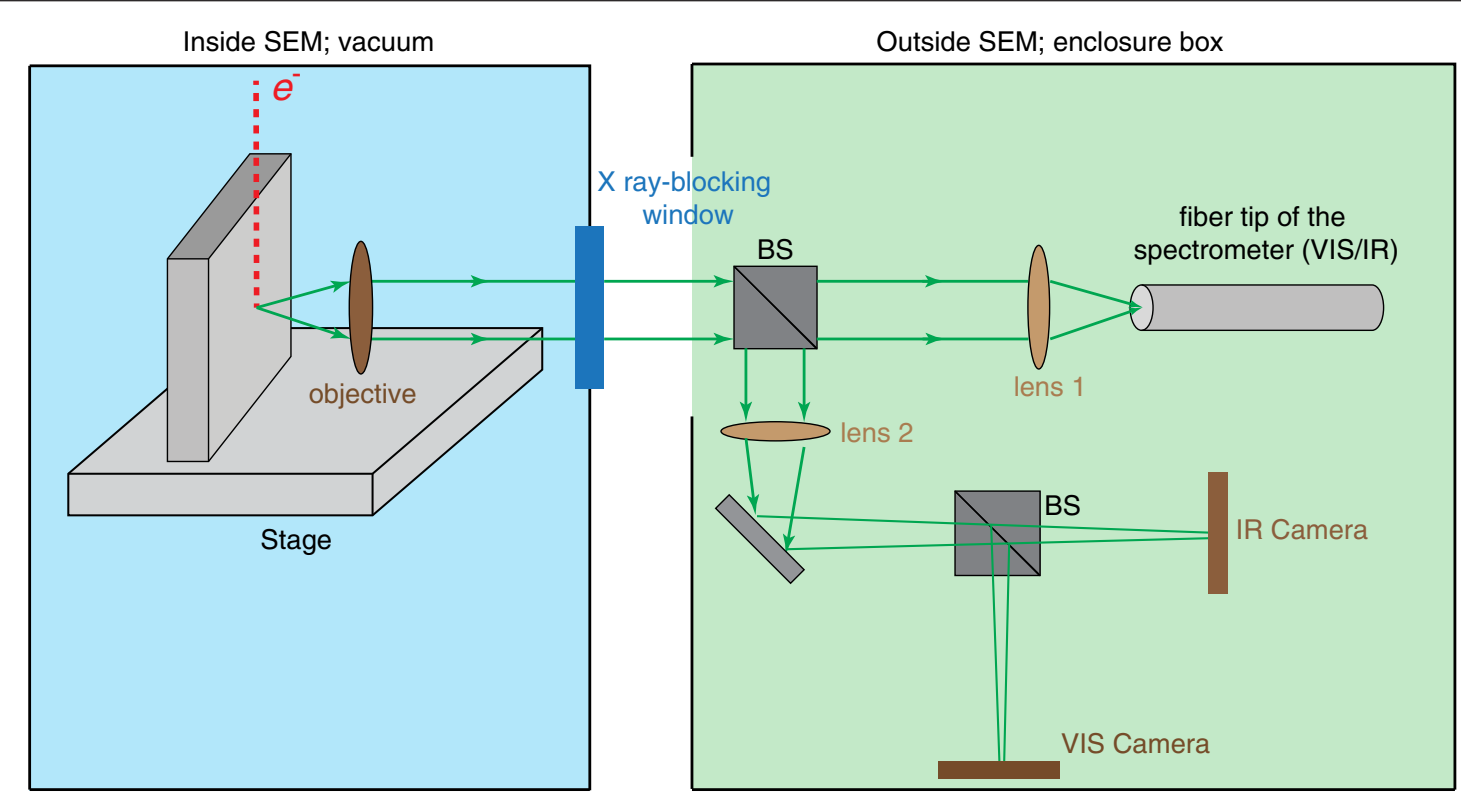

FIG. 1. Illustration of the experimental setup. The left part (shaded in blue) describes the modified stage inside the vacuum chamber of the SEM. The sample is held so that its surface is almost parallel to the path of the electron beam. The emitted light is collected by an objective, directing the emitted light into an enclosure box holding the rest of the optical setup. The right part (shaded in green) describes the optical collection setup, having a beam splitter (BS) that splits the optical beam to a fiber tip collector leading to a spectrometer (right of BS) and two CCD cameras (below the BS). Right before the spectrometer fiber, we place a polarizer. Lens 2 is used to image the surface of the sample on two CCDs. We choose between them with a flip mirror. We change among three CCDs: a visible CCD, a less-sensitive but color-visible CCD, and a near-infrared CCD.

hemisphere above the surface [48-51]. However, angular $\mathrm{CL}$ only records the emission angle and does not provide a direct way to detect the origin of the emitted light; this is particularly important in cases where light propagates away from the local excitation spot before it is emitted. In contrast, instead of using a parabolic mirror, our system uses an objective lens to resolve the precise location from which light is emitted. This collection method is particularly useful for cases where electrons are striking at a glancing angle to the sample, enabling the observation of a large area interaction, as is present in the Smith-Purcell effect.

The samples studied below are based on a silver surface patterned with periodic structures, which are either 1D or two-dimensional (2D) periodic structures (i.e., gratings or plasmonic crystals). The patterns are created by either depositing silver structures on the surface of a planar silver film or by milling the patterns into the surface of a film via a focused ion beam (FIB). When launching free electrons parallel to these structures, we find that they always exhibit effects of collective light emission in the form of Smith-Purcell radiation: Free electrons passing parallel to the periodic structure excite effective dipoles positioned with the periodicity of the structure (period $a$ ). The dipoles oscillate with a relative phase delay that depends on the velocity of the electron $v$ (or its velocity divided by the speed of light, $\beta=v / c$ ). The dipoles emit radiation that interferes constructively in the far field, creating the conventional Smith-Purcell effect [11]. The radiation at wavelength $\lambda$ is only emitted along specific angles $\theta$ (measured relative to the electron direction of motion), for different diffraction orders $m$, following the formula [11]

$$
\lambda=\frac{a}{m}\left(\frac{1}{\beta}-\cos (\theta)\right)
$$

Figure 2 presents spectrum measurements at angles near the normal to the surface, for electron velocities between $\beta=0.27(20 \mathrm{kV})$ and $\beta=0.21(12 \mathrm{kV})$, from a $1 \mathrm{D}$ grating with periodicity $a=200 \mathrm{~nm}$. For these parameters, we only measure the first diffraction order $m=1$ appearing in between 750 and $950 \mathrm{~nm}$ [Fig. 2(b)]. Our experimental results show that the peak of the spectrum shifts to longer wavelengths for electrons with lower speed $(\beta)$, which agrees with Eq. (1). For a quantitative comparison, we overlay the theoretical predictions of the emission peaks under different accelerating voltages with dashed lines, which agree well with our experimental results (see Methods section).

Apart from the above collective electron excitation, another possibility for light-emitting interactions of the electrons with our structures are those through localized excitations. These include the excitation of dipoles in localized resonances and other kinds of CL-related effects [18,47-51], some coming from atomic transitions of 


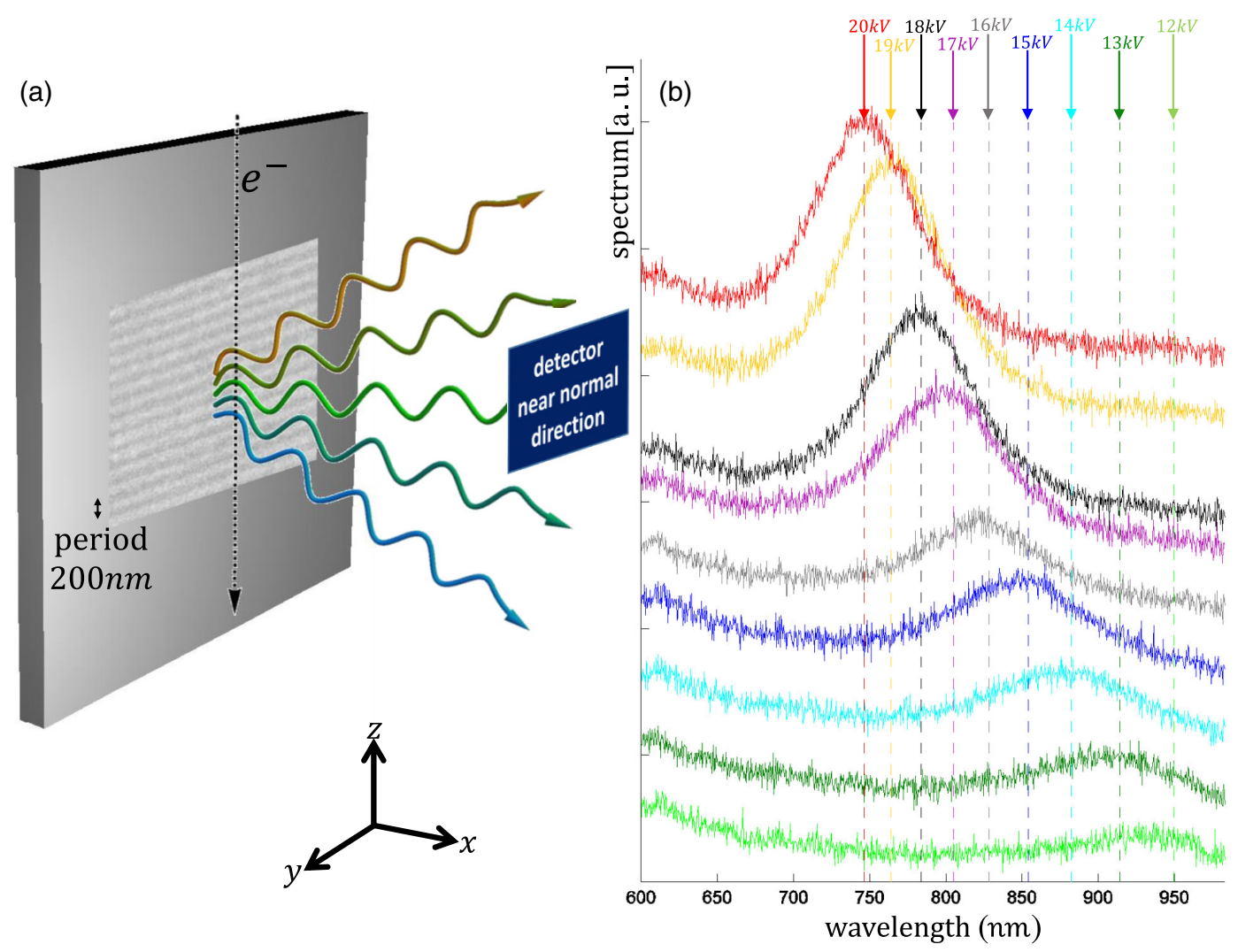

FIG. 2. The conventional Smith-Purcell effect. (a) Illustration of the interaction presenting the electrons as a black dashed arrow, passing near a grating (which is shown by its real SEM image). The colored wiggly arrows represent the emitted Smith-Purcell radiation. (b) Measured spectra for different kinetic energies. (The measurements are displaced vertically for clarity.) The dashed vertical lines are calculated according to the conventional Smith-Purcell theory, with the color corresponding to the same kinetic energies.

excited electronic states [e.g., any sample emits at least some amount of CL, as can be seen, in this case, by the light-blue emission outside the frame marking the grating position in Figs. 3(c) and 3(d)]. This shows that our setup not only detects the spectrum but also images the surface showing where the light is coming from. Figure 3 presents the imaging of the surface of two samples - one presented in Figs. 3(c) and 3(d) for electrons with kinetic energies of $13 \mathrm{kV}$ and $20 \mathrm{kV}$, respectively, and the other presented in Fig. 3(e) for kinetic energy of $20 \mathrm{kV}$. The first sample shows enhanced emission from both the top edge of the pattern [Fig. 3(c)] and from a defect line in the middle of the sample [Fig. 3(d)], while the second sample shows inhibited emission from a defect line in the middle of the sample [Fig. 3(e)]. Why is one sample showing enhanced emission and the other inhibited emission? The first sample [Figs. 3(c) and 3(d)] has a grating deposited on top, while the second sample [Fig. 3(e)] has a grating milled into the surface. For collective excitations such as the conventional Smith-Purcell effect, these methods are equivalent since a deposited grating and a milled grating are equivalent (the deposited material is silver, just like the sample surface, and the silver thickness of $500 \mathrm{~nm}$ is practically infinite for light or plasmon propagation, emission, and absorption).
Nevertheless, the difference between depositing the grating and milling it becomes apparent when considering the interaction with nearly tangential electrons. This is because the cross section for the interaction is different exactly around the edge of the pattern and the line defect (see Fig. 3 for an illustration). Therefore, the emission of light through local excitations, whose intensity is proportional to the local flux of electrons, will increase [Figs. 3(c) and 3(d)] or decrease [Fig. 3(e)], respectively. Notice that no conventional electromagnetic simulation can capture the entire effect since emission from local excitations (e.g., CL) cannot be modeled by Maxwell's equations alone [33]. Instead, this effect requires some model of the electronic transitions in the material. Nonetheless, as we show below in Figs. 4(d) and 5 and the accompanying discussion, much insight can be gained on the emission from local excitations by collecting spectra and considering the emission of an antenna excited by an effective dipole. Figure $\mathrm{S} 1$ in Ref. [52] shows, additionally, that the emission from the edge and line defect contains different spectral components than the background conventional Smith-Purcell emission.

We now turn our attention to the interaction of free electrons with a 2D plasmonic crystal with disorder that has a periodicity in both the direction of the electron motion 


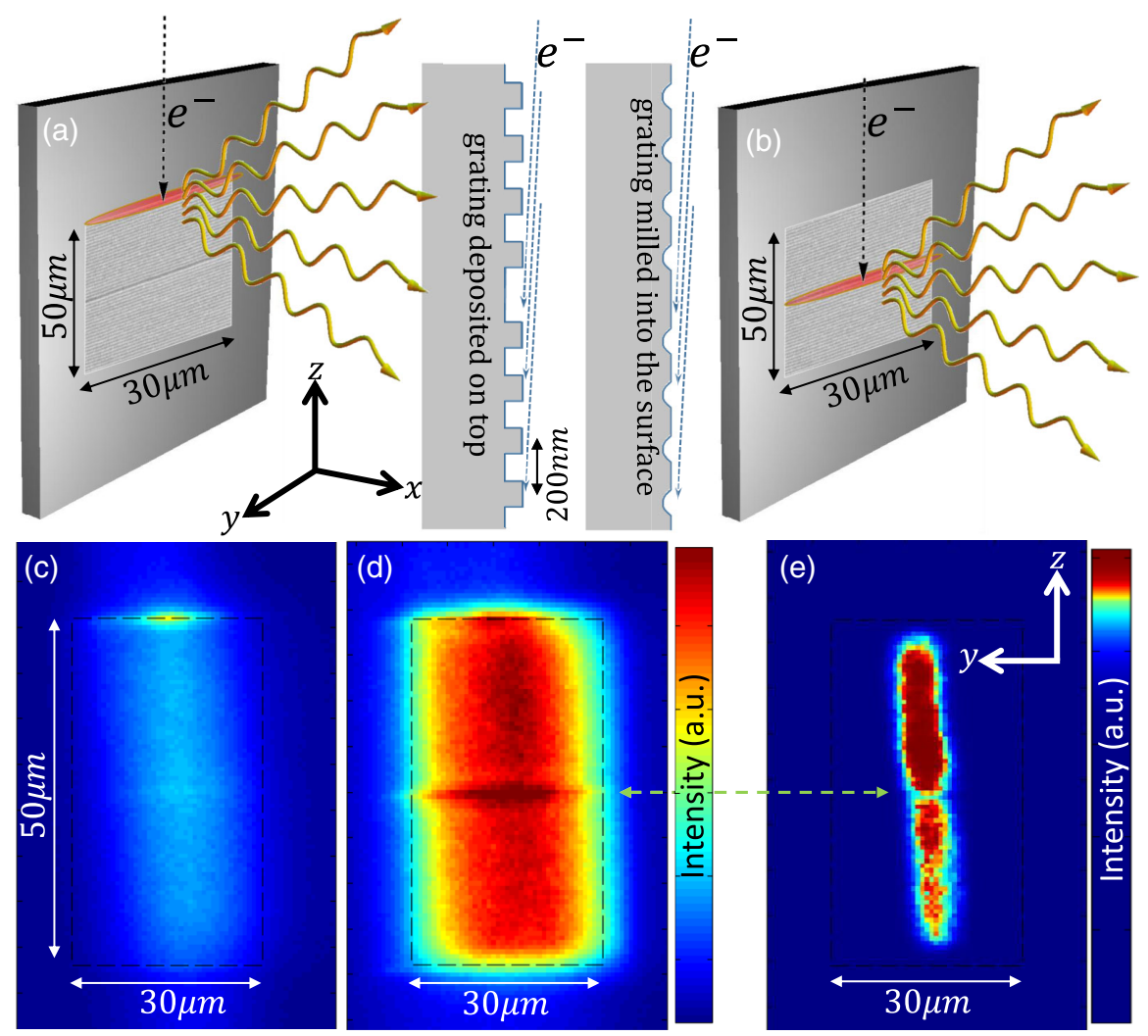

FIG. 3. Direct imaging of enhanced and inhibited emission from line defect. (a,b) Illustration of the electron beam (dashed black arrow) interacting with line defects fabricated in the samples. (c-e) False color optical images of the light emission from the samples showing a clear enhancement (c,d) or inhibition (e) of the emission from the edge of the periodic structure and from the line defect. The samples are fabricated by either electron beam lithography with silver deposition $(\mathrm{c}, \mathrm{d})$ or by focused ion beam milling of trenches into a silver film (e). These techniques result in different cross sections to the electrons interacting with the gratings, as illustrated by the grating profiles between (a) and (b), leading to enhanced and inhibited emission, respectively. The electrons' kinetic energy is $13 \mathrm{kV}$ and $20 \mathrm{kV}$ in (c) and (d,e), respectively.

(average $145 \mathrm{~nm}$ ) and perpendicular to it $(575 \mathrm{~nm})$. Here, even the spectral signature contains, simultaneously, two mechanisms of emitted radiation-the collective effect of conventional Smith-Purcell radiation [Figs. 4(a) and 4(c)] and the localized plasmonic resonance [Figs. 4(b) and 4(d)]. Interestingly, as our measurements in Fig. 4 show, these mechanisms can be very well separated by polarization filtering.

The conventional Smith-Purcell radiation emitted in directions near normal to the surface is polarized primarily in the direction of the electron motion $z$ (since the electron-photon coupling goes like $\vec{v} \cdot \vec{E}$ ). At the same time, the emission from the localized plasmonic resonances is polarized perpendicular to the direction of electron motion $y$, owing to the shape of the silver "rods" comprising the plasmonic structure. These rods are elongated along the $y$ axis since the periodicity in the $y$ axis $(575 \mathrm{~nm})$ is much larger than the periodicity in the $z$ axis $(145 \mathrm{~nm})$. Therefore, the induced dipole is oriented along the $y$ axis, causing the emitted radiation to be polarized along the same axis. This logic is fully consistent with our simulations of the structure (Fig. 5), showing the induced dipole in the field distribution ([Fig. 5(d)], and finding the spectral resonance at about $730 \mathrm{~nm}$ [Figs. 5(a) and 5(b)], fully agreeing with our measurements (Fig. 4(d)).

Finally, we analyze the surprising robustness to disorder that we find in the measurements of the plasmonic crystal. Disorder occurs naturally in the fabrication process of our plasmonic crystal (because of the large aspect ratio between the rods' height and width, their small size, and the liftoff process-see Methods section). As we will see below, despite strong disorder [SEM image shown in Fig. 6(e)], some intrinsic order still protects the Smith-Purcell effect, even though it is an inherent result of the periodicity.

Figure 5(a) shows the resonances that would be expected to appear in light-reflection measurements in our disordered plasmonic structure, had it been fabricated as exactly periodic. The resonance on the right, at about $730 \mathrm{~nm}$, appears because of the localized plasmonic resonance in the individual silver rods comprising the plasmonic structure, as is shown by field distribution in Fig. 5(d). Fully agreeing with this prediction, the 730-nm resonance appears in our measurements [Fig. 4(d)]. In addition to this peak, the 

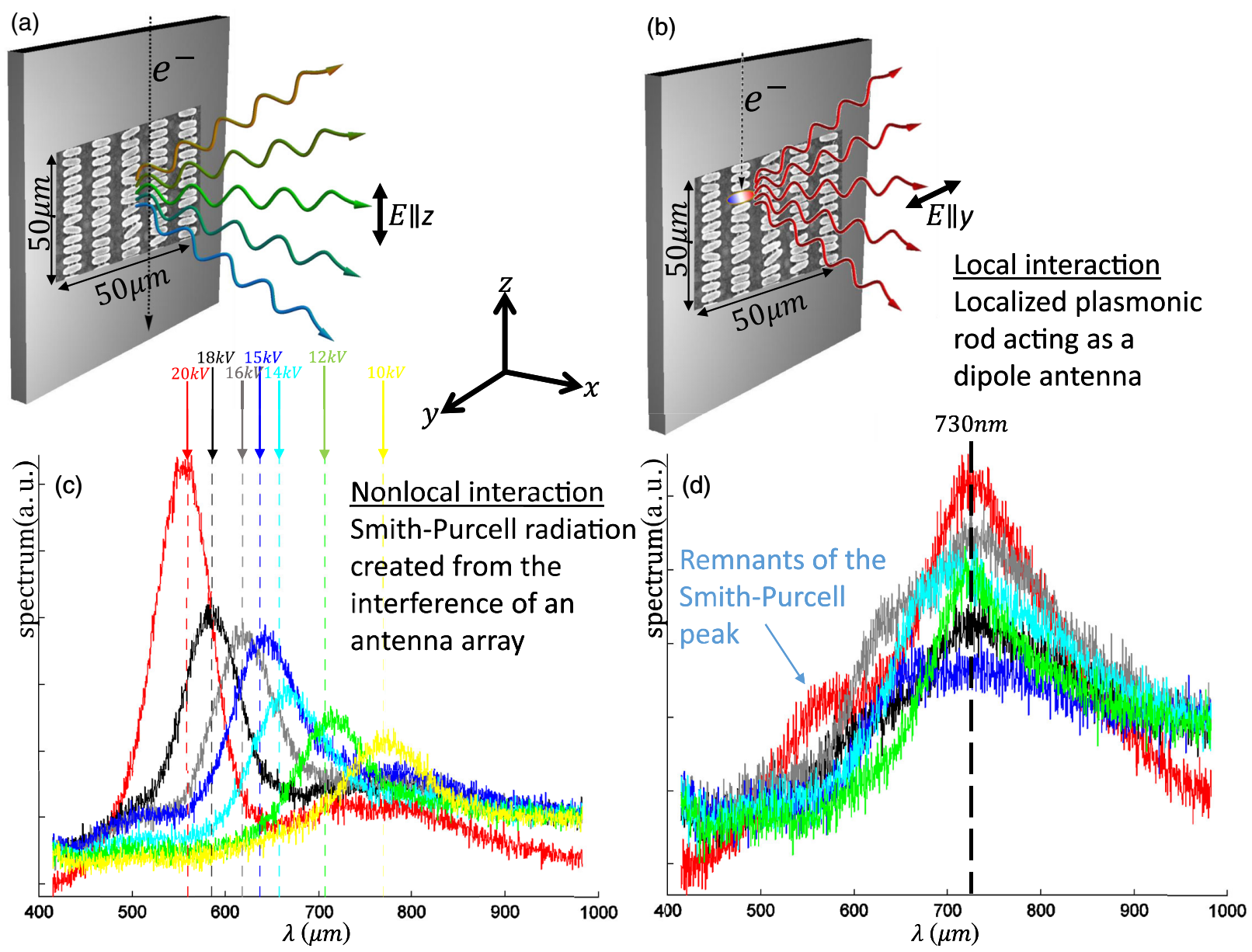

FIG. 4. Collective and resonant effects in the interaction of free electrons with a disordered plasmonic structure. (a) Illustration of the Smith-Purcell emission from the 2D plasmonic crystal with strong disorder. (b) Illustration of the emission from the localized plasmonic resonance of a single silver rod. In both (a) and (b), the electron beam is plotted with a dashed black line, and the emitted light is drawn as wiggly arrows. The semiperiodic structure itself is the SEM image of the real pattern. (c) Measuring the spectra for different kinetic energies with a polarizer that transmits light polarized along the $z$ axis. This shows a very good match to conventional Smith-Purcell theory [Eq. (1)], marked by dashed lines in the color corresponding to the same kinetic energies. (d) Measuring the spectrum with a polarizer that transmits light polarized along the $y$ axis. This shows a single plasmonic resonance at $730 \mathrm{~nm}$, agreeing with simulations (see Fig. 6). Note that small features from the spectral peaks of (c) can be seen in (d) and vice versa. This is explained by the conventional Smith-Purcell radiation not being entirely $z$ polarized in this structure, in addition to imperfections in the polarization filtering. For comparison of the scales, the red peak in (c) is 2.4 times more intense than the red peak in (d).

Smith-Purcell peak appears clearly, as shown in Fig. 4(a). This is surprising since the conventional Smith-Purcell [Eq. (1)] is the result of a collective excitation that is intuitively expected to be sensitive to disorder. The appearance of the Smith-Purcell peak is even more peculiar when recalling that the conventional Smith-Purcell effect itself can actually also be described as a Bloch mode (albeit a leaky mode [53]), which is typically destroyed even by weak disorders.

We believe that the reason for the robustness of the Smith-Purcell effect is a long-range order that exists in the disordered plasmonic structure simultaneously with strong local disorder. This long-range order is due to the fabrication process placing the rods in pivot positions, which are periodic in design. Around these pivot positions, the rods experience short-range disorder shifting them away from perfect periodicity to their actual final positions. The process creates a distribution that, despite having wide variance in positions (and a wide variance in nearest-neighbor distance), still retains long-range order with an average periodicity close to the designed $145 \mathrm{~nm}$.

To bring a quantitative test of the robustness, we use image processing to identify the positions of the silver rods (in the SEM image): The automatically identified positions are marked with red circles in Fig. 6(e) (the figure only shows a small subsection of a much larger area for which we performed the image processing). In these retrieved positions, we position oscillating dipoles and calculate the far-field interference pattern, shown in Figs. 6(a) and 6(c). These are compared to the simulation 

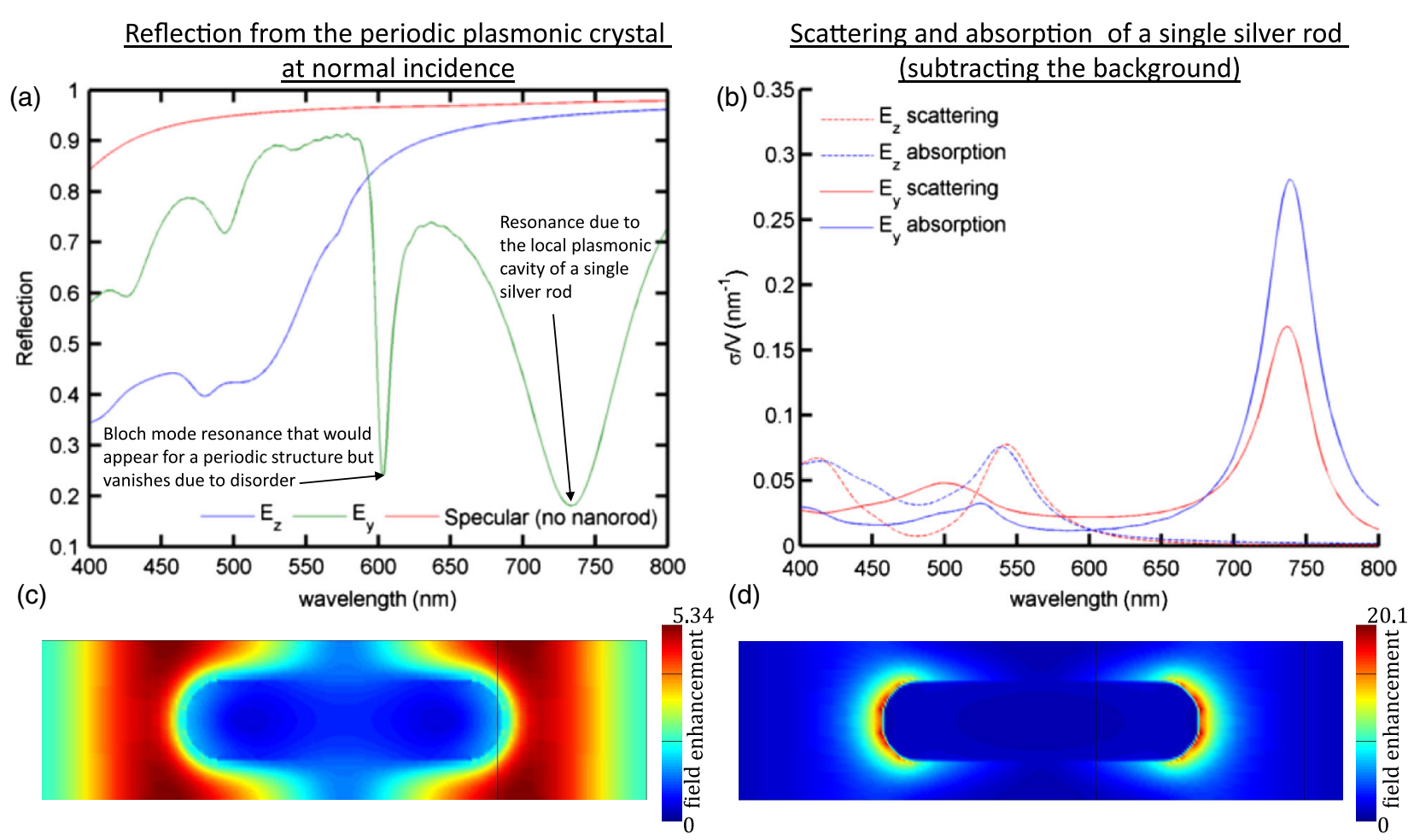

FIG. 5. The localized plasmonic resonance of a single silver rod. (a) The reflection of a normal incidence beam polarized along the $z(y)$ axis in blue (green), simulated for an exactly periodic plasmonic crystal, similar to the structure in Figs. 4 and 6. For reference, we plot in red the reflection from a silver surface without nanorods. (b) Total absorption (blue) and scattering (red) from a single silver nanorod (subtracting the background by simulating a silver surface without any feature). The results for $z(y)$-polarized incidence are plotted by a dashed (solid) curve. (c) Electric-field amplitude (absolute value) for the resonance at about $600 \mathrm{~nm}$. The field distribution makes it clear that this is a Bloch mode resonance. Indeed, this resonance disappears when simulating the structure with absorbing boundary conditions in (b) (perfectly matched layers-PMLs) instead of periodic boundary conditions in (a). (d) Electric-field amplitude (absolute value) of the localized plasmonic resonance at about $730 \mathrm{~nm}$, which appears in both (a) and (b) as expected.

of an ordered array in Figs. 6(b) and 6(d). The simulation shows that despite its large disorder, the Smith-Purcell radiation pattern should still appear, as indeed happens experimentally.

This robustness is closely related to other physical phenomena in solid-state physics and optics, described by the DebyeWaller factor, and the Ruze formula (e.g., Refs. [54,55]). Both of these quantities are essentially averages of oscillating exponentials with Gaussian-distributed phases, which represent the intensity of the far-field radiation pattern in the direction normal to the surface:

$$
\int_{-\infty}^{+\infty} e^{i \theta} e^{-\left(\theta^{2} / 4 \sigma^{2}\right)} \propto e^{-\sigma^{2}} .
$$

Here, the peak power of the far field always scales as $e^{-\sigma^{2}}$, where $\sigma$ is the deviation of the phase distribution. In our case, this random phase distribution is correlated to the random offsets of the rod positions. The key point is that disorder, as long as it is random, lowers the peak intensity rather than moving it or broadening it, which gives us the robustness we see here. This averaging applies to the Smith-Purcell effect because the electron interaction with a disordered structure induces an array of radiating dipoles that has a disordered phase as a function of position; the far-field radiation from these dipoles is given by a sum of exponentials as in the Debye-Waller effect. This explains why the Smith-Purcell spectral peak is observed in the disordered plasmonic structure.

In summary, our work demonstrates the exciting potential of a modified electron microscope with both spectral and spatial resolving capabilities for making new physics observable. The SEM is a broadly available tool that is user-friendly and relatively easy to modify. It gives various opportunities to combine it with the flexibility of optical setups and enables a field of opportunities in observing phenomena involving the interaction of electrons and photons.

Methods.-We began fabrication of the 1D sample (Figs. 2 and 3) by depositing $500 \mathrm{~nm}$ of $\mathrm{Ag}$ on a silicon slab using an electron-beam evaporator. Milled structures were made using a Raith FIB machine working at $35 \mathrm{kV}$ with a current of $45 \mathrm{pA}$ and a line dose of $20,000 \mathrm{uC} / \mathrm{cm}$. Deposited structures were produced by a standard electron beam lithography: PMMA 950K A4 resist was spin coated at $4000 \mathrm{rpm}$ and baked on a hot plate at $120^{\circ} \mathrm{C}$ for 

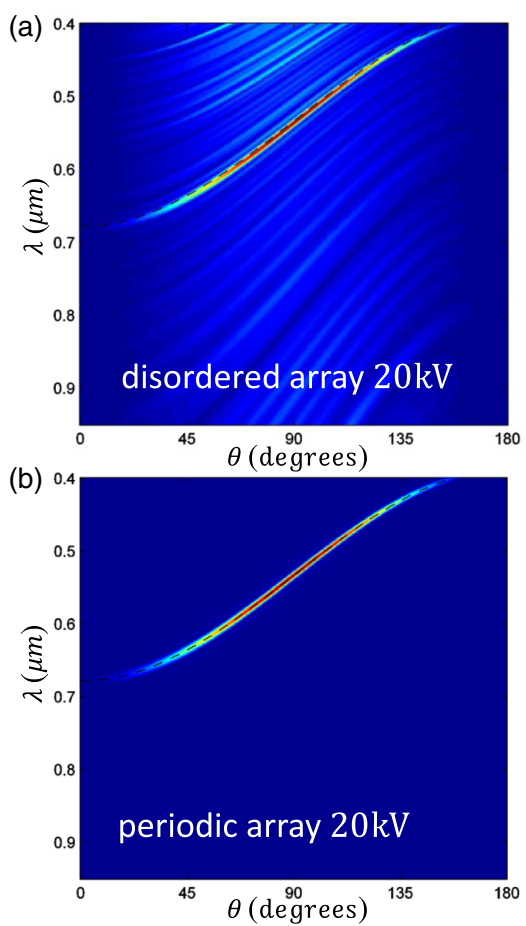

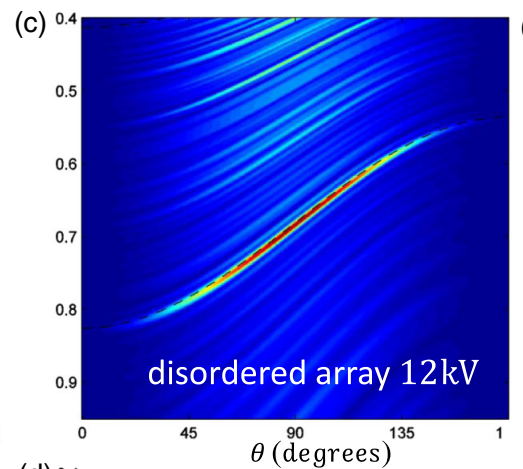

(d)

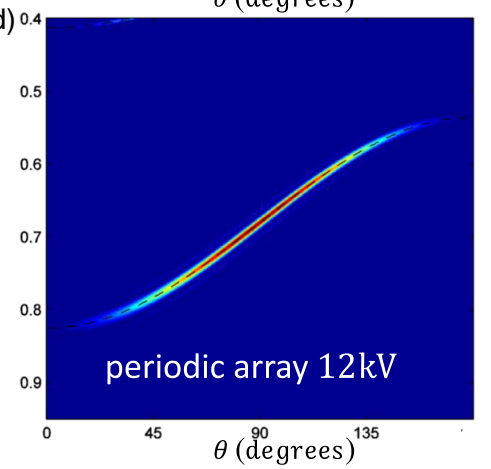

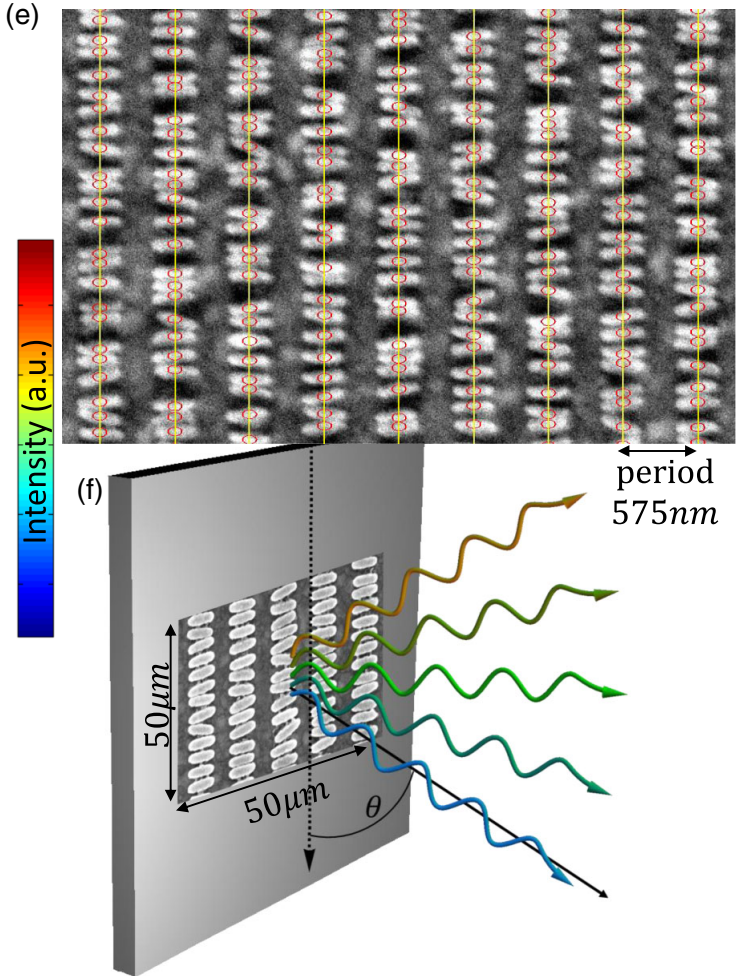

FIG. 6. Collective contribution from a disordered structure. Simulation of the Smith-Purcell dispersion relation, plotting the far-field Poynting vector along the radial direction as a function of the polar angle in a disordered array $(\mathrm{a}, \mathrm{c})$ and a periodic array $(\mathrm{b}, \mathrm{d})$ with a pitch of $145 \mathrm{~nm}$, which is the mean value for the disordered array. We choose the positions of the disordered array by image processing of the SEM image (e) - retrieving the positions of the rods marked by red circles overlaying the image. The illustration of the light emission is presented in (f), marking the polar angle of emission $\theta$. The noise robustness and the dispersion relation are independent of the azimuthal angle (taken to be zero in the simulation here).

5 minutes. The structures were then written using a Raith IonLine electron beam lithography machine operating at $10 \mathrm{kV}$ with a current of $8 \mathrm{pA}$ and line dose of $320 \mathrm{pC} / \mathrm{cm}$. After development, $50 \mathrm{~nm}$ of $\mathrm{Ag}$ were evaporated onto the sample, whereafter the sample was placed in acetone to complete liftoff.

Fabrication of the 2D sample (Figs. 4 and 6) was again completed by electron beam lithography, on a silicon slab with $150 \mathrm{~nm}$ of evaporated Ag. The beam operated at $20 \mathrm{kV}$ with a current of $120 \mathrm{pA}$ and a line dose of $600 \mathrm{pC} / \mathrm{cm}$. The grating height was again set to $50 \mathrm{~nm}$ of $\mathrm{Ag}$ by evaporation.

The SEM used for the experiment was a JEOL JSM6010LA. Its energy spread at the gun exit was in the range 1.5 to $2.5 \mathrm{eV}$ for the range of acceleration voltages considered in this paper. A Nikon TU Plan Fluor 10x objective with a numerical aperture (NA) of 0.30 was used to collect light from the area of interest. The monochrome images were taken using a Hamamatsu CCD, while the color images were taken using a Thor Labs CCD Camera $(1024 \times 768$ resolution). The spectrometer used was an Acton Research Corporation Spectrapro 2300i with a lownoise Princeton Instruments Pixis 400 CCD.
The theoretical predictions of the Smith-Purcell peaks in Figs. 2(b) and 4(c) were found through Eq. (1) by using the angle as our only fitting parameter. The angles found were $\theta=93^{\circ}$ and $\theta=100^{\circ}$ for Figs. 2(b) and 4(c), respectively; both indicate collection near the direction normal to the surface. The deviation from the normal is due to the need to tilt the sample to allow the electron beam to interact with the relevant area of the sample.

Reflection spectra, scattering and absorption cross sections, and mode profiles were obtained using a threedimensional (3D) finite-time-finite-difference simulation (Lumerical). Reflection spectra of the periodic structure were obtained via a 3D simulation in Lumerical. Plane waves were normally incident onto the structure. Periodic boundary conditions were imposed. The reflection was calculated via the surface integration of the Poynting vector behind the plane-wave source. Mode profiles were obtained by Fourier transforming the time-domain signal into the frequency domain using appropriate apodization to eliminate irrelevant transient responses.

Scattering and absorption cross sections of a single nanorod were obtained using the 3D total-field-scatteredfield (TFSF) simulation in Lumerical. A TFSF plane-wave 
source was placed around the scatterer. Perfectly matched layers (PMLs) were placed surrounding the simulation region. In-plane mirror symmetries were exploited to reduce calculation complexity. A surface integral on the Poynting vector $\vec{S}$ surrounding the scatterer was calculated to extract the scattering cross section:

$$
\sigma_{\mathrm{sca}}=\frac{1}{I_{0}} \iint \hat{n} \cdot \vec{S} d a,
$$

where $\hat{n}$ is the unit vector pointing out of the surface, and $I_{0}$ is the source intensity. We note that the scattering cross section includes the excitation of surface plasmons and free-space photons. A volume integral on the loss per unit volume inside the scatterer was calculated to extract the absorption cross section:

$$
\sigma_{\mathrm{abs}}=\frac{1}{I_{0}} \iiint \frac{1}{2} \operatorname{Im}(\epsilon) \omega|E|^{2} d V
$$

where $|E|$ is the absolute value of the amplitude of the electric field, $\omega$ is the frequency, and $\epsilon$ is the (complex) permittivity of the material (silver).

The simulations of the Smith-Purcell effect in periodic and disordered structures in Fig. 6 were performed by placing effective dipoles at the rods' positions and calculating the electromagnetic fields as the superposition of these dipoles. We used the far-field expressions of a single dipole:

$$
\begin{aligned}
& \vec{E} \propto \frac{1}{\mathrm{r}} \sin \theta e^{i(\omega / c) r-i \omega t} \hat{\theta}, \\
& \vec{B} \propto \frac{1}{\mathrm{r}} \sin \theta e^{i(\omega / c) r-i \omega t} \hat{\phi},
\end{aligned}
$$

where $\hat{\theta}$ is the polar unit vector, $\hat{\phi}$ is the azimuthal unit vector, and $r$ is the radial distance. The resulting Poynting vector from a set of dipoles was calculated and plotted (Fig. 6) at the far field (at a fixed radius $r \rightarrow \infty$ ) as a function of the polar angle. The dipoles were placed on the surface at positions $z_{i}$ along the path of the electron (moving along the $\hat{z}$ direction), where the relative phase delay between the dipoles depends on the velocity $v$ of the electron,

$$
\vec{S} \propto \frac{1}{\mathrm{r}^{2}} \sin ^{2} \theta\left|\sum_{i} e^{i(\omega / c)\left|\vec{r}-z_{i} \hat{z}\right|} e^{i(\omega / v) z_{i}}\right|^{2} \hat{r} .
$$

\section{ACKNOWLEDGMENTS}

We thank Dr. Mark Knight and Dr. Kevin F. MacDonald for their advice and Dr. Giorgio Adamo for the helpful discussions. The work was supported by the US Army Research Laboratory and the US Army Research Office through the Institute for Soldier Nanotechnologies (Contract No. W911NF-13-D-0001), and by the Israel Science Foundation, Grant No. 1310/13 and the
German-Israeli Project cooperation (DIP). J. J. L. is supported in part by a NSF Graduate Research Fellowship under award No. 1122374. J. J. L. and Y. Y. were supported in part by the MRSEC Program of the National Science Foundation under award No. DMR-1419807. The research of I. K. was partially supported by the Seventh Framework Programme of the European Research Council (FP7-Marie Curie IOF) under Grant agreement No. 328853MC-BSiCS.

[1] P. A. Cherenkov, Visible Emission of Clean Liquids by Action of $\gamma$ Radiation, Dokl. Akad. Nauk SSSR 2, 451 (1934).

[2] S. Liu, P. Zhang, W. Liu, S. Gong, R. Zhong, Y. Zhang, and M. Hu, Surface Polariton Cherenkov Light Radiation Source, Phys. Rev. Lett. 109, 153902 (2012).

[3] S. Xi, H. Chen, T. Jiang, L. Ran, J. Huangfu, B.-I. Wu, J. A. Kong, and M. Chen Experimental Verification of Reversed Cherenkov Radiation in Left-Handed Metamaterial, Phys. Rev. Lett. 103, 194801 (2009).

[4] P. Genevet, D. Wintz, A. Ambrosio, A. She, R. Blanchard, and F. Capasso, Controlled Steering of Cherenkov Surface Plasmon Wakes with a One-Dimensional Metamaterial, Nat. Nanotechnol. 10, 804 (2015).

[5] C. Luo, M. Ibanescu, S. G. Johnson, and J. D. Joannopoulos, Cerenkov Radiation in Photonic Crystals, Science 299, 368 (2003).

[6] F. J. García de Abajo, Multiple Excitation of Confined Graphene Plasmons by Single Free Electrons, ACS Nano 7, 11409 (2013).

[7] S. Liu, C. Zhang, M. Hu, X. Chen, P. Zhang, S. Gong, T. Zhao, and R. Zhong, Coherent and Tunable Terahertz Radiation from Graphene Surface Plasmon Polaritons Excited by an Electron Beam, Appl. Phys. Lett. 104, 201104 (2014).

[8] I. Kaminer, M. Mutzafi, A. Levy, G. Harari, H. H. Sheinfux, S. Skirlo, J. Nemirovsky, J. D. Joannopoulos, M. Segev, and M. Soljačić, Quantum Čerenkov Radiation: Spectral Cutoffs and the Role of Spin and Orbital Angular Momentum, Phys. Rev. X 6, 011006 (2016).

[9] I. Kaminer, Y. T. Katan, H. Buljan, Y. Shen, O. Ilic, J. J. López, L. J. Wong, J. D. Joannopoulos, and M. Soljačić, Quantum Čerenkov Effect from Hot Carriers in Graphene: An Efficient Plasmonic Source, Nat. Commun. 7, 11880 (2016).

[10] A. Friedman, A. Gover, G. Kurizki, S. Ruschin, and A. Yariv Spontaneous and Stimulated Emission from Quasifree Electrons, Rev. Mod. Phys. 60, 471 (1988).

[11] S. J. Smith and E. M. Purcell, Visible Light from Localized Surface Charges Moving across a Grating, Phys. Rev. 92, 1069 (1953).

[12] P. M. Van den Berg, Smith-Purcell Radiation from a Point Charge Moving Parallel to a Reflection Grating, J. Opt. Soc. Am. 63, 1588 (1973).

[13] A. Gover, P. Dvorkis, and U. Elisha, Angular Radiation Pattern of Smith-Purcell Radiation, JOSA B 1, 723 (1984). 
[14] N. Horiuchi et al. Exotic Radiation from a Photonic Crystal Excited by an Ultrarelativistic Electron Beam, Phys. Rev. E 74, 056601 (2006).

[15] G. Adamo, K. F. MacDonald, Y. H. Fu, C.-M. Wang, D. P. Tsai, F. J. García de Abajo, and N. I. Zheludev Light Well: A Tunable Free-Electron Light Source on a Chip, Phys. Rev. Lett. 103, 113901 (2009).

[16] G. Adamo, J. Y. Ou, J. K. So, S. D. Jenkins, F. De Angelis, K. F. MacDonald, E. Di Fabrizio, J. Ruostekoski, and N. I. Zheludev Electron-Beam-Driven Collective-Mode Metamaterial Light Source, Phys. Rev. Lett. 109, 217401 (2012).

[17] K. Tantiwanichapan, X. Wang, A. K. Swan, and R. Paiella, Graphene on Nanoscale Gratings for the Generation of Terahertz Smith-Purcell Radiation, Appl. Phys. Lett. 105, 241102 (2014).

[18] J.-K. So, F. Javier García de Abajo, K. F. MacDonald, and N. I. Zheludev Amplification of the Evanescent Field of Free Electrons, ACS Photonics 2, 1236 (2015).

[19] N. Yamamoto, F. J. Garcia de Abajo, and V. Myroshnychenko, Interference of Surface Plasmons and Smith-Purcell Emission Probed by Angle-Resolved Cathodoluminescence Spectroscopy, Phys. Rev. B 91, 125144 (2015).

[20] P. Zhang, L. K. Ang, and A. Gover, Enhancement of Coherent Smith-Purcell Radiation at Terahertz Frequency by Optimized Grating, Prebunched Beams, and Open Cavity, Phys. Rev. ST Accel. Beams 18, 020702 (2015).

[21] Y. Takeda and I. Matsui, Laser Linac with Grating, Nucl. Instrum. Methods 62, 306 (1968).

[22] K. Mizuno, J. Pae, T. Nozokido, and K. Furuya, Experimental Evidence of the Inverse Smith-Purcell Effect, Nature (London) 328, 45 (1987).

[23] E. A. Peralta et al. Demonstration of Electron Acceleration in a Laser-Driven Dielectric Microstructure, Nature (London) 503, 91 (2013).

[24] J. Breuer and P. Hommelhoff, Laser-Based Acceleration of Nonrelativistic Electrons at a Dielectric Structure, Phys. Rev. Lett. 111, 134803 (2013).

[25] D. Bar-Lev and J. Scheuer, Plasmonic Metasurface for Efficient Ultrashort Pulse Laser-Driven Particle Acceleration, Phys. Rev. ST Accel. Beams 17, 121302 (2014).

[26] S. Yamaguti, J. I. Inoue, O. Haeberlé, and K. Ohtaka, Photonic Crystals Versus Diffraction Gratings in SmithPurcell Radiation, Phys. Rev. B 66, 195202 (2002).

[27] K. Yamamoto et al. Observation of Millimeter-Wave Radiation Generated by the Interaction between an Electron Beam and a Photonic Crystal, Phys. Rev. E 69, 045601 (2004).

[28] T. Ochiai and K. Ohtaka, Electron Energy Loss and SmithPurcell Radiation in Two- and Three-Dimensional Photonic Crystals, Opt. Express 13, 7683 (2005).

[29] J. R. M. Saavedra, D. Castells-Graells, and F. J. García de Abajo, Smith-Purcell Radiation Emission in Aperiodic Arrays, Phys. Rev. B 94, 035418 (2016).

[30] S. Chuang and J. Kong, Enhancement of Smith-Purcell Radiation from a Grating with Surface-Plasmon Excitation, JOSA A 1, 672 (1984).

[31] T. Ochiai and K. Ohtaka, Relativistic Electron Energy Loss and Induced Radiation Emission in Two-Dimensional Metallic Photonic Crystals. I. Formalism and Surface Plasmon Polariton, Phys. Rev. B 69, 125106 (2004).
[32] N. Yamamoto, K. Araya, and F. J. García de Abajo, Photon Emission from Silver Particles Induced by a High-Energy Electron Beam, Phys. Rev. B 64, 205419 (2001).

[33] W. Li, W. Liu, and Q. Jia, Investigation on the Special Smith-Purcell Radiation from a Nano-Scale Rectangular Metallic Grating, AIP Adv. 6, 035202 (2016).

[34] R. H. Ritchie, Plasma Losses by Fast Electrons in Thin Films, Phys. Rev. 106, 874 (1957).

[35] C. J. Powell and J. B. Swan, Origin of the Characteristic Electron Energy Losses in Aluminum, Phys. Rev. 115, 869 (1959).

[36] R. B. Pettit, J. Silcox, and R. Vincent, Measurement of Surface-Plasmon Dispersion in Oxidized Aluminum Films, Phys. Rev. B 11, 3116 (1975).

[37] P. E. Batson, Damping of Bulk Plasmons in Small Aluminum Spheres, Solid State Commun. 34, 477 (1980).

[38] M. Bashevoy, F. Jonsson, A. Krasavin, N. Zheludev, Y. Chen, and M. I. Stockman, Generation of Traveling Surface Plasmon Waves by Free-Electron Impact, Nano Lett. 6, 1113 (2006).

[39] J. Van Wijngaarden, E. Verhagen, A. Polman, C. Ross, H. Lezec, and H. Atwater, Direct Imaging of Propagation and Damping of Near Resonance Surface Plasmon Polaritons Using Cathodoluminescence Spectroscopy, Appl. Phys. Lett. 88, 221111 (2006).

[40] F. J. García de Abajo, Optical Excitations in Electron Microscopy, Rev. Mod. Phys. 82, 209 (2010).

[41] S. Nie and S. R. Emory, Probing Single Molecules and Single Nanoparticles by Surface-Enhanced Raman Scattering, Science 275, 1102 (1997).

[42] K. A. Willets and R. P. Van Duyne, Localized Surface Plasmon Resonance Spectroscopy and Sensing, Annu. Rev. Phys. Chem. 58, 267 (2007).

[43] S. John, Strong Localization of Photons in Certain Disordered Dielectric Superlattices, Phys. Rev. Lett. 58, 2486 (1987).

[44] T. Schwartz, G. Bartal, S. Fishman, and M. Segev, Transport and Anderson Localization in Disordered Two-Dimensional Photonic Lattices, Nature (London) 446, 52 (2007).

[45] J. Billy, V. Josse, Z. Zuo, A. Bernard, B. Hambrecht, P. Lugan, D. Clément, L. Sanchez-Palencia, P. Bouyer, and A. Aspect Direct Observation of Anderson Localization of Matter Waves in a Controlled Disorder, Nature (London) 453, 891 (2008).

[46] L. J. Wong, I. Kaminer, O. Ilic, J. D. Joannopoulos, and M. Soljačić Towards Graphene Plasmon-Based FreeElectron Infrared to X-Ray Sources, Nat. Photonics 10, 46 (2016).

[47] J.-K. So, K.F. MacDonald, and N. I. Zheludev, Fiber Optic Probe of Free Electron Evanescent Fields in the Optical Frequency Range, Appl. Phys. Lett. 104, 201101 (2014).

[48] T. Coenen, E. J. R. Vesseur, A. Polman, and A. F. Koenderink, Directional Emission from Plasmonic Yagi-Uda Antennas Probed by Angle-Resolved Cathodoluminescence Spectroscopy, Nano Lett. 11, 3779 (2011).

[49] R. Sapienza, T. Coenen, J. Renger, M. Kuttge, N. F. Van Hulst, and A. Polman, Deep-Subwavelength Imaging of the Modal Dispersion of Light, Nat. Mater. 11, 781 (2012). 
[50] C. I. Osorio, T. Coenen, B. J. Brenny, A. Polman, and A. F. Koenderink, Angle-Resolved Cathodoluminescence Imaging Polarimetry, ACS Photonics 3, 147 (2015).

[51] T. Coenen, B. J. Brenny, E. J. Vesseur, and A. Polman, Cathodoluminescence Microscopy: Optical Imaging and Spectroscopy with Deep-Subwavelength Resolution, MRS Bull. 40, 359 (2015).

[52] Supplemental Material at http://link.aps.org/supplemental/ 10.1103/PhysRevX.7.011003, for additional figure showing the spectral signature of the localized plasmonic resonances.

[53] F. Monticone and A. Alu, Leaky-Wave Theory, Techniques, and Applications: From Microwaves to Visible Frequencies, Proc. IEEE 103, 793 (2015).

[54] J. Ruze, Antenna Tolerance Theory-A Review, IEEE Proc. 54, 633 (1966).

[55] G. Grosso and G. P. Parravicini, Solid State Physics (Elsevier Science, London, 2000). 